STUDI

FRANCESI

\section{Studi Francesi}

Rivista quadrimestrale fondata da Franco Simone

174 (LVIII | III) | 2014

Varia

\title{
Aa. Vv., Lectures de Stendhal. "Le Rouge et le Noir", sous la direction de Xavier Bourdenet
}

Michel Arrous

\section{(2) OpenEdition}

Journals

\section{Édition électronique}

URL : http://journals.openedition.org/studifrancesi/1543

DOI : 10.4000/studifrancesi. 1543

ISSN : 2427-5856

Éditeur

Rosenberg \& Sellier

\section{Édition imprimée}

Date de publication : 1 novembre 2014

Pagination : 615-616

ISSN : 0039-2944

\section{Référence électronique}

Michel Arrous, «Aa. Vv., Lectures de Stendhal. "Le Rouge et le Noir", sous la direction de Xavier

Bourdenet », Studi Francesi [En ligne], 174 (LVIII | III) | 2014, mis en ligne le 01 novembre 2014, consulté le 18 septembre 2020. URL : http://journals.openedition.org/studifrancesi/1543 ; DOI : https://doi.org/ 10.4000/studifrancesi. 1543

Ce document a été généré automatiquement le 18 septembre 2020.

\section{(c)}

Studi Francesi è distribuita con Licenza Creative Commons Attribuzione - Non commerciale - Non opere derivate 4.0 Internazionale. 


\title{
Aa. Vv., Lectures de Stendhal. "Le Rouge et le Noir", sous la direction de Xavier Bourdenet
}

\author{
Michel Arrous
}

\section{RÉFÉRENCE}

AA. VV., Lectures de Stendhal. "Le Rouge et le Noir", sous la direction de Xavier BOURDENET, Presses Universitaires de Rennes, 2013, pp. 266.

1 Agrégation oblige: en 2013, le Rouge a fait l'objet de nombreuses études et, inévitablement, de nombreuses redites, ce qui n'empêcha sans doute pas les agrégatifs de tirer parti de cette profusion. Dans le cas présent, c'est la réception actuelle du roman qui a été choisie, d'abord en privilégiant son inscription historique, puis en s'intéressant à la représentation des personnages, aux différents régimes du romanesque et, enfin, à la poétique de l'énonciation. Dans sa présentation, "Le Rouge et le Noir" en 1830: "Billet gagnant», roman monstre (pp. 11-35), Xavier BOURDENET évoque le moment d'émergence de ce roman politique dont les premiers lecteurs ont immédiatement saisi le caractère paradoxal en stigmatisant la noirceur de Julien, ou en discutant la vraisemblance d'un récit jugé hors-normes, lequel finira par être normalisé sinon canonisé. François VANOOSTHUYSE ("Le Rouge et le Noir" et 1830, pp. 39-52) voit dans l'absence de références à la révolution de Juillet «une véritable difficulté herméneutique» que bien des lecteurs négligent, plus sensibles au fait que Julien incarnerait d'une certaine façon la Révolution ou une "classe insurrectionnelle». Se demander si la «chronique de 1830 » est bien un roman de 1830 , ou si le roman n'est pas l'œuvre de quelqu'un qui n'a pas vu venir les Trois Glorieuses, est fort discutable; plus convaincante, à coup sûr, l'assertion suivante: «le fait révolutionnaire n'était pas compatible avec le scénario du Rouge, ni avec sa signification d'ensemble ni avec la signification particulière de son personnage principal». Si Julien n'a rien d'un 
révolutionnaire de 1830, il est néanmoins au centre d'un roman «engagé», voire «militant». C'est d'ailleurs ce qu'illustre l'exemple du séminaire choisi par Yves ANSEL (Le séminaire, l'enfer et Frilair, pp. 53-68) pour mettre en évidence le rôle crucial de la religion. Miroir «partiel et partial», le roman nous renvoie une image biaisée de «la peu ragoûtante cuisine ecclésiastique». Marta CARAION (Représentations matérielles et fonctions imaginaires des objets dans "Le Rouge et le Noir", pp. 69-85) aborde un aspect rarement évoqué dans la critique rougiste qui s'est portant intéressée à l'activité descriptive. Au lieu de décrire les objets, Stendhal préfère en montrer l'utilisation - il est vrai que la nécessité de décrire une échelle ne s'impose pas! À partir d'une liste d'ailleurs incomplètement exploitée (l'habit noir, les échelles, le vase du Japon, la vieille épée) est proposée une poétique de l'objet dont la fonctionnalité littéraire n'est qu'esquissée.

2 Dans la deuxième partie («Personnel romanesque et usages du monde») l'accent est mis sur le prétendu roman de l'ambitieux, la représentation du masculin ou les modèles dont s'inspirent les personnages et qui assurent leur individualisation. Jacques-David EBGUY (Un «défaut de ligne droite». "Le Rouge et le Noir", un singulier roman d'apprentissage, pp. 89-103) relève les aspects qui contreviennent au récit d'apprentissage. De l'examen du contenu narratif, il ressort qu'une série de coups de force et de hasards modifient sans cesse la situation du "parvenu qui ne parvient à rien» (R. Judrin) et qui, loin d'aller de l'avant, ne cesse de regarder vers son passé. Le Rouge n'est pas un roman d'apprentissage (le Bildungsroman "promeut la réconciliation de l'homme et du monde»), mais plutôt un «roman désorienté» dans lequel Stendhal aurait paradoxalement exploité toutes les potentialités du roman d'apprentissage. Les contributions de Jean-Marie Roulin (Masculin et pouvoir dans "Le Rouge et le Noir", pp. 105-120), Daniel MAIRA (L'énergie virilisante de la Renaissance dans "Le Rouge et le Noir", pp. 121-136) et François KERLOUÉGAN (Un roman désincarné? Le corps dans "Le Rouge et le Noir", pp. 137-152) ont des préoccupations communes: la dimension genrée des personnages, la représentation du corps et de la masculinité, avec ses idéologies et son monopole sur le pouvoir, et de la masculinisation, chez Mathilde. Muriel BAssou relit le roman à l'aune de l'amitié, en distinguant ses catégories et ses représentations, de l' "amitié amoureuse» au "commerce armé», entre l'amitié en province et l'amitié à Paris. Fondée sur le désintéressement, l'amitié selon Stendhal n'est pas celle qu'une longue tradition a définie (Représentations de l'amitié dans "Le Rouge et le Noir", pp. 153-169).

Il y a dans la "chronique de 1830» quelques échappées vers le romanesque. C'est le filon que suivent Catherine MARIETTE (Présence du romanesque, pp. 173-186), Pascale AURAIXJONCHÈRE (Le cœur mangé et la tête coupée: les régimes du romanesque, pp. 187-199) et Philippe BERTHIER (Intermezzo Buffo, pp. 201-205). Tous trois montrent que le roman se nourrit d'une succession de scènes réalistes et romanesques. Il peut s'agir d'un romanesque sentimental, avec Mme de Rênal, héroïque, avec Mathilde, ou du romanesque de la légende médiévale, et même de l'intrusion brève mais efficace en la personne du chanteur Geronimo, bouffée romanesque qui chasse en un instant la «nausée politique».

Dans la dernière partie consacrée à des problèmes de poétique, Pierre LAFORGUE revient sur le statut des italiques du "Rouge et le Noir" (pp. 209-224), lues comme un «ensignement du réel de 1830». Dans la typologie proposée - italique de convention, de citation, d'inflexion, d'énonciation - les deux derniers usages sont les plus intéressants. Le discours italique «doublerait [-il] le texte en romain du roman»? Son usage, au même titre que les intrusions de l'auteur et les autres éléments paratextuels, participe à une 
stratégie énonciative qu'on ne peut réduire à une seule définition tant il est instable. Ce manque de cohérence serait un effet de la crise de la représentation à l'âge romantique. L'étude des structures narratives permet de repérer, non sans les subtils distinguos qu'affectionne Boris LYON-CAEN (Les pulsations du récit, pp. 225-235), trois modes de configuration, tension et relâchement, répétition, préparation et rôle des modèles, qui viennent perturber la linéarité d'un récit dont l'ambition réaliste est revendiquée. Pour sa part, Maria scotT, qui reprend le problème du point de vue dans (la lecture de) "Le Rouge et le Noir" (pp. 236-248), montre à partir de quelques exemples que le roman est caractérisé par une «instabilité perspectiviste» telle que le lecteur peut hésiter devant le sens à attribuer aux événements, quand il ne se retrouve pas devant une véritable opacité ou des lectures incompatibles. Dans Figuration romanesque: profils psychologiques et partis pris poétiques (pp. 249-260), Philippe JOUSSET, tout en mettant en évidence une stylistique de la ligne brisée (analyser/estomper), mène une très fine réflexion sur le style de vie et la caractérisation des personnages: à Mme de Rênal, «unie et constante», et la seule à être idéalisée, s'opposent Julien, «presque toujours dissocié d'avec soi, en perpétuels anticipations et retards», et Mathilde qui semble créée pour «tester l'étendue des réactions de Julien». 